\title{
Zur Situation der Infektionen des Menschen durch enterohämorrhagische Escherichia coli (EHEC) in Deutschland 1997
}

\author{
Von J. Bockemühl, H. Karch und H. Tschäpe
}

\section{Zusammenfassung}

1997 wurden in den Laboratorien der Autoren insgesamt 101 Fälle von hämolytisch-urämischem Syndrom (HUS) durch Erregerisolierung (69 Fälle) oder durch Nachweis spezifischer anti-LPS IgM-Antikörper gegen die EHEC-O-Gruppe 157 (32 Fälle) gesichert. Hinzu kommen 171 Enteritisfälle durch EHEC und 13 Isolate von Ausscheidern oder unbekannter Herkunft. Ergebnisse einer laborgestützten Sentinelstudie bei ambulanten Durchfallpatienten sowie Untersuchungen an hospitalisierten Kindern mit Darmsymptomatik ergaben einen EHEC-Nachweis in 2-3 \% der Fälle. EHEC erwiesen sich somit zusammen mit Campylobacter hinter den Salmonellen als zweithäufigste Enteritiserreger. Das Erregerspektrum war charakterisiert durch eine zunehmende Ausweitung, vor allem zu Lasten der O-Gruppe 157, die 1997 nur noch in 65,2\% der HUS- und $29,2 \%$ der Enteritisfälle beteiligt war. Neben den bereits in den Vorjahren gehäuft nachgewiesenen EHEC O157 (O157:H7, O157:H-), O26 (O26:H11, O26:H-), O111 (O111:H2, O111:H-) und O103 (O103:H2, O103:H-) wurde 1997 EHEC O145 (O145:H28, O145:H-) dritthäufigster HUS-Erreger. Darüber hinaus waren $10,1 \%$ der HUS- und $39,8 \%$ der Enteritisfälle durch insgesamt 25 Serovare und 27 nicht typisierbare oder Rauhstämme mit inkompletten Virulenzmustern beteiligt. Eine Unterscheidung in EHEC (eae und EHEC-bly-positiv) bzw. STEC oder VTEC-Stämme (inkomplettes Pattern von Virulenzmarkern) ist deshalb für Infektionen des Menschen nicht sinnvoll. Im Hinblick auf die Shigatoxin-Typen wiederholte sich die Beobachtung des Vorjahres, daß Shigatoxin-2-produzierende Stämme in signifikanter Weise mit HUS assoziiert sind $(97,1 \%)$, während von Enteritisfällen in 39,8 \% Isolate erhalten wurden, die nur Shigatoxin 1 bildeten. Die 1997 beobachtete weitere Ausbreitung von EHEC in der Umwelt gibt zur Sorge Anlaß und erfordert die intensive Ermittlung der Infektionsursachen bei EHEC-Infektionen des Menschen, um rechtzeitig geeignete Maßnahmen zum Schutz der Bevölkerung und der Verbraucher ergreifen zu können.

\section{Summary}

Human infections caused by enterohaemorrhagic Escherichia coli (EHEC) in Germany 1997

In 1997 , a total of 101 cases of haemolytic uraemic syndrome (HUS) were identified in the authors' laboratories by positive culture (69 cases) or detection of IgM antibodies to O157 LPS (32 cases). Additionally, 171 cases of EHEC enteritis and 19 strains from healthy subjects or unknown clinical conditions were confirmed. Results from a laboratory-based sentinel study in non-hospitalized diarrhoeal patients as well as a study in hospitalized children with intestinal symptoms revealed an EHEC isolation rate of $2-3 \%$. Thus, together with Campylobacter but less frequently than Salmonella, EHEC actually figure on the second rank of bacterial agents causing human diarrheal illness. The expanding pattern of serovars observed since several years, continued in 1997: EHEC O157 was identified in only $65.2 \%$ of HUS and $29.2 \%$ of enteritis cases. Besides O157 (O157:H7, O157:H-), O26 (O26:H11, O26:H-), O111 (O111:H2, O111:H-), and O103 (O103:H2, O103:H-), serogroup $\mathrm{O} 145(\mathrm{O} 145: \mathrm{H} 28, \mathrm{O} 145: \mathrm{H}-)$ emerged and was identified in $8.7 \%$ of HUS patients. Furthermore, from 10.1 $\%$ of HUS and $39.8 \%$ of enteritis cases a total of 25 serovars and further 27 non-typable or serologically rough strains with an incomplete pattern of virulence factors (eae, EHEC haemolysin) were isolated. It thus appears useless to distinguish between EHEC (complete virulence pattern) and STEC or VTEC (incomplete virulence pattern) as far as human infections are concerned. Analysis of shiga toxin patterns confirmed our previous observation that HUS is significantly associated with shiga toxin $2(97.1 \%)$ whereas strains producing shiga toxin 1 only, are mainly associated with enteritis $(38.9 \%)$. The expanding pattern of EHEC strains in human infections and the continuous spread of the organisms in the environment are an issue of serious concern, demanding for intensive investigation of the various routes of transmission in order to prompt adequate measures for effective health protection of the population and the consumers.

\section{Einleitung}

Infektionen des Menschen durch enterohämorrhagische Escherichia coli (EHEC) haben 1997 in der Öffentlichkeit, bei den Behörden sowie seitens des öffentlichen Gesundheitsdienstes eine deutlich gesteigerte Beachtung gefunden. Nach der Publikation eines Merkblatts für Ärzte über EHEC-Infektionen [1] wurden als erster Schritt zum Aufbau einer effektiveren Surveillance Empfehlungen der Arbeitsgruppe
VTEC/EHEC des RKI und des BgVV sowie der Fachgruppe "Gastrointestinale Infektionen« der Deutschen Gesellschaft für Hygiene und Mikrobiologie (DGHM) vorgelegt, die eine vorläufige Falldefinition für EHEC-bedingte Erkrankungen, eine Indikationsliste zur mikrobiologischen Untersuchung und Mindestanforderungen an das diagnostische Vorgehen im klinisch-mikrobiologischen Labor zum Inhalt haben [2] Nachdem in Bayern zum 1. 4. 1996 die
Meldepflicht für EHEC-Infektionen verordnet worden ist und dort innerhalb eines Jahres 300 Nachweise beim Menschen festgestellt wurden (1.4.1996 bis 31. 3. 1997) [3], haben sich bis September 1997 weitere 13 Bundesländer bereit erklärt, dem RKI die Zahlen der gemeldeten EHEC-bedingten Erkrankungen im Rahmen wöchentlicher Meldungen zu übermitteln und darüber hinaus Einzelfallerhebungen mittels eines vom RKI erarbeiteten Fragebogens durchzu- 
führen. Bis September 1997 wurden auf dieser Grundlage bundesweit 275 EHEC-Erkrankungen mitgeteilt [2] Wenn auch die erfaßten Infektionen sicher nicht repräsentativ sind, stellen sie eine deutliche Verbesserung der Datensituation im Vergleich zu den Vorjahren dar.

Ziel der vorliegenden Arbeit ist, in Fortführung vorangegangener Berichte $[4$, 5], in erster Linie die Analyse der erregerspezifischen Merkmale, um Trends der Erregerdynamik aufzuzeigen und den Nachweis bestimmter Virulenzmarker bzw. ihrer Kombinationen in Relation zu Krankheitsbildern zu bewerten. Die Studie umfaßt Isolate von Fällen, die 1997 am Institut für Hygiene und Mikrobiologie der Universität Würzburg und in der Abteilung Bakteriologie des Hygiene Instituts Hamburg isoliert bzw. dem NRZ am RKI Wernigerode zugesandt worden sind.

\section{Ergebnisse}

\section{Inzidenz}

Wie in Tabelle 1 dargestellt ist, wurden 1997 insgesamt 101 Fälle von hämolytisch-urämischem Syndrom (HUS) durch Erregerisolierung (69 Fälle) oder durch Nachweis von IgM-Antikörpern gegen das Lipopolysaccharidantigen von EHEC O157 (32 Fälle) gesichert. Diese Zahl entspricht praktisch der Zahl bestätigter HUS-Erkrankungen des Vorjahres (1996: 96 Fälle).

Dagegen war die Zahl bestätigter Enteritisfälle mit 171 Patienten weit mehr als doppelt so hoch wie 1996 (63 Fälle). Dies ist sicher nicht nur Folge der offensichtlichen Ausbreitungstendenz der EHEC-Bakterien, sondern auch der zunehmend besseren Kenntnis bei den behandelnden Ärzten, die EHEC häufiger in ihre diagnostischen Überlegungen einbeziehen. Sechs Stämme stammten von gesunden Ausscheidern, und bei 13 Isolaten war wegen fehlender Angaben eine Zuordnung zu einem Krankheitszustand nicht möglich.

Im Rahmen einer laborgestützten Sentinelstudie wiesen Prager u. Mitarb. [6] bei $2,9 \%$ (111 von 3835 ) ambulanter Enteritispatienten einen hohen Anteil von EHEC-Bakterien nach; die Studie ist in diesem Heft ausführlich beschrieben. Dagegen bestätigte sich bei hospitalisierten Kindern der UniversitätsKinderklinik Würzburg mit Darmsymptomatik, die seit 1991 mit gleichbleibender Methodik auf EHEC unter-

Tabelle 1: Serovare und Virulenzmarker bei EHEC-Stämmen vom Menschen, Deutschland 1997

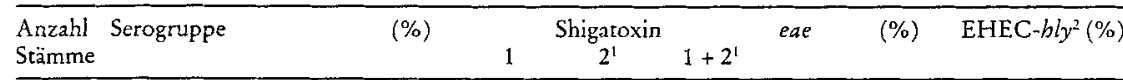

HUS-Fälle (bakteriologisch 69 Fälle, serologisch [Anti-O157-LPS-Antikörper] 32 Fälle, gesamt 101 Fälle)

\begin{tabular}{|c|c|c|c|c|c|c|c|}
\hline 45 & $\mathrm{O} 157: \mathrm{H}^{\prime}, \mathrm{H}^{-}$ & $(65,2 \%)$ & 0 & 43 & 2 & $45(100,0 \%)$ & $45(100,0 \%)$ \\
\hline 7 & $\mathrm{O} 26: \mathrm{H} 11, \mathrm{H}^{-}$ & $(10,1 \%)$ & 0 & 5 & 2 & $7(100,0 \%)$ & $7(100,0 \%)$ \\
\hline 6 & $\mathrm{O} 145: \mathrm{H}^{-}$ & $(8,7 \%)$ & 1 & 5 & 0 & $6(100,0 \%)$ & $6(100,0 \%)$ \\
\hline 3 & $\mathrm{O} 103: \mathrm{H} 2, \mathrm{H}^{-}$ & $(4,4 \%)$ & 0 & 3 & 0 & $3(100,0 \%)$ & $2(66,6 \%)$ \\
\hline 1 & $\mathrm{O} 111: \mathrm{H}^{-}$ & $(1,5 \%)$ & 0 & 0 & 1 & $1(100,0 \%)$ & $1(100,0 \%)$ \\
\hline 7 & übrige Serovare ${ }^{3}$ & $(10,1 \%)$ & 1 & 5 & 1 & $4(57,1 \%)$ & $4(57,1 \%)$ \\
\hline & & & 2 & 61 & 6 & $66 \quad(95,7 \%)$ & $65(94,2 \%)$ \\
\hline
\end{tabular}

Enteritis-Fälle $(n=171)$

\begin{tabular}{|c|c|c|c|c|c|c|c|}
\hline 50 & $\mathrm{O} 157: \mathrm{H}, \mathrm{H}^{-}$ & $(29,2 \%)$ & 3 & 41 & 6 & $49 \quad(98,0 \%)$ & $48 \quad(96,0 \%)$ \\
\hline 22 & $\mathrm{O} 26: \mathrm{H}_{11}, \mathrm{H}^{-}$ & $(12,9 \%)$ & 13 & 6 & 3 & $19(86,4 \%)$ & $20(90,9 \%)$ \\
\hline 13 & $\mathrm{O} 103: \mathrm{H} 2, \mathrm{H} 18, \mathrm{H}^{-}$ & $(7,6 \%)$ & 12 & 1 & 0 & $11(84,6 \%)$ & $9(69,2 \%)$ \\
\hline 10 & O111: $\mathrm{H}^{-}$ & $(5,8 \%)$ & 6 & 0 & 4 & $10(100,0 \%)$ & $10(100,0 \%)$ \\
\hline 8 & $\mathrm{O} 145: \mathrm{H} 28, \mathrm{H}^{-}$ & $(4,7 \%)$ & 2 & 6 & 0 & $8(100,0 \%)$ & $8(100,0 \%)$ \\
\hline 68 & übrige Serovare ${ }^{+}$ & $(39,8 \%)$ & 32 & 26 & 10 & $15(22,1 \%)$ & $47 \quad(69,1 \%)$ \\
\hline 171 & & & 68 & 80 & 23 & $112(65,5 \%)$ & $142(83,0 \%)$ \\
\hline 1 & Citrobacter freundii & & 0 & 1 & 0 & 0 & 0 \\
\hline
\end{tabular}

Ausscheider und unbekannt $(\mathrm{n}=19)$

\begin{tabular}{|c|c|c|c|c|c|c|c|}
\hline 11 & $\mathrm{O} 157: \mathrm{H} 7, \mathrm{H}^{-}$ & 0 & 10 & 1 & $11(100,0 \%)$ & 10 & $(90,9 \%)$ \\
\hline 8 & übrige Serovare $^{5}$ & 6 & 0 & 2 & $1 \quad(12,5 \%)$ & 5 & $(62,5 \%)$ \\
\hline 19 & & 6 & 10 & 3 & $12(63,2 \%)$ & 15 & $(78,9 \%)$ \\
\hline
\end{tabular}

1 einschl. Shigatoxin 2-Varianten

2 genorypischer Nachweis des EHEC-Hämolysins

3 gesamt 6 Serovare und 1 nicht typisierbarer Stamm

4 gesamt 25 Serovare einschl. unbeweglicher Varianten und 27 nichr O-typisierbare oder Rauhstämme

5 gesamr 3 Serovare und 5 nichr O-rypisierbare oder Rauhstämme

sucht werden, eine seit 1994 gleichbleibende Inzidenz: EHEC wurden bei neun von 405 Durchfallpatienten nachgewiesen, entsprechend einer Positivrate von $2,2 \%(1994: 2,8 \%, 1995: 2,5 \%$, 1996: 2,4\%). Aus den genannten Zahlen ist $\mathrm{zu}$ folgern, daß bei klinischer Schwere des Krankheitsbildes, das eine bakteriologische Stuhluntersuchung nach sich zieht, EHEC mit einer relativen Häufigkeit von 2 bis $3 \%$ beteiligt sind. Sie liegen damit zusammen mit Campylobacter und hinter den Salmonellen an zweiter Stelle nachgewiesener Enteritiserreger.

Die größte Krankheitshäufung ereignete sich in Niedersachsen. Dort wurden zwischen Januar und August 1997 allein 21 Fälle von HUS, z. T. zusätzlich mit zerebraler Symptomatik, bei Kindern festgestellt [7].

\section{Erregerspektrum und Toxintypen}

Die seit 1994 beobachtete Ausweitung des Erregerspektrums setzte sich auch 1997 fort. So ging vor allem der prozentuale Anteil der Serogruppe O157 bei den Erkrankungen weiter zurück. Bei den 69 bakteriologisch bestätigten HUS-Fällen wurden nur noch 65,2\% durch die Serovare O157:H7 und $\mathrm{O} 157: \mathrm{H}^{-}$hervorgerufen (45 Fälle; 1994/95: 78,4 \%, 1996: 70,5 \%; Tab. 1). Stämme der Serogruppe O26 (O26:H11, $\left.\mathrm{O} 26: \mathrm{H}^{-}\right)$sind seit vier Jahren mit etwa $10 \%$ die zweithäufigsten Erreger, während die ebenfalls seit langem bereiligten Typen O111:H2 und $\mathrm{O} 111: \mathrm{H}^{-}$ leicht zurückgegangen sind. Nachdem EHEC der O-Gruppe 103 (O103:H2, O103: $\left.\mathrm{H}^{-}\right) 1996$ erstmals beim HUS und vor allem auch bei der Enteritis größere Bedeutung erlangt haben, wurden 1997 
Tabelle 2: Assoziation von Shigatoxintypen mit HUS- und Enteritisfällen in Deutschland, $1997(\mathrm{n}=240)$

\begin{tabular}{|c|c|c|c|c|}
\hline Shigatoxin & HUS & $(\%)$ & Enteritis & $(\%)$ \\
\hline Stx 1 & 2 & $(2,9 \%)$ & 68 & $(39,8 \%)$ \\
\hline Stx $2 *$ & 61 & \multirow{2}{*}{$(97,1 \%)$} & 80 & $(46,8 \%)$ \\
\hline Stx $1+2 *$ & 6 & & 23 & $(13,5 \%)$ \\
\hline gesamt & 69 & & 171 & \\
\hline
\end{tabular}

* einschl. Shigatoxin 2-Varianten

Stämme des Serovars O145: $\mathrm{H}^{-}$mit $8,7 \%$ dritthäufigste HUS-Erreger. Mit sieben Fällen wurden weitere $10 \%$ der HUS-Fälle durch andere Serovare hervorgerufen (O4: $\mathrm{H}^{-}, \mathrm{O} 23: \mathrm{H}^{-}, \mathrm{O} 55: \mathrm{H} 6$, O112: $\mathrm{H}^{-}, \mathrm{O} 118: \mathrm{H}^{-}, \mathrm{O} 165: \mathrm{H}^{-}$und ein nicht typisierbarer Stamm, ONT: $\mathrm{H}^{-}$).

Bei den Enteritisfäller war EHEC O157 nur noch mit $29,2 \%$ beteiligt (1994/95: 43,2 \%, 1996: 31,3\%), gefolgt, z. T. mit rückläufiger Tendenz, von O26, O103, und O111 (Tab. 1). Stämme der neu in Erscheinung getretenen Serogruppe O145 (O145:H28, O145: $\mathrm{H}^{-}$) belegten den vierten Platz $(4,7 \%)$. Besonders bemerkenswert ist die Zunahme der übrigen Serovare: 68 bakteriologisch gesicherte Fälle $(39,8 \%)$ wurden durch 25 weitere Typen einschließlich unbeweglicher Varianten sowie durch 27 nicht typisierbare oder Rauhstämme hervorgerufen. Die Entwicklung des bei zwei Enteritisfällen durch EHEC O103 erstmals beobachteten Serovars O103:H18 sollte beobachtet werden. Auch muß daran erinnert werden, daß Citrobacter freundii gelegentlich Shigatoxin 2 produzieren kann; 1997 wurde wieder ein Fall von Enteritis durch diese Keime festgestellt.

Das die EHEC im engeren Sinne determinierende, Intimin-kodierende eaeGen war bei 66 von 69 HUS-Isolaten vorhanden $(95,7 \%)$, während bei den Enteritis-Isolaten dieses Merkmal nur bei 112 von 171 Stämmen $(65,5 \%)$ nachgewiesen wurde (Tab. 1). Ähnlich verhielt es sich mit dem Gen des EHECHämolysins (EHEC-bly), das bei $94,2 \%$ der HUS-Stämme und bei $83 \%$ der Enteritis-Isolate nachweisbar war (Tab. 1). Hierbei ist allerdings zu berücksichtigen, daß nicht alle Stämme, die dieses Gen beherbergen, das Hämolysin auch exprimieren.

Wie bereits im Vorjahr [5], muß auf die offensichtlich unterschiedliche pathogenetische Bedeutung der Shigatoxin (Stx)-Typen hingewiesen werden. Stämme, die nur Shigatoxin 1 bilden, waren bei nur 2,9\% der HUS-Fälle, aber bei $39,8 \%$ der Enteritisfälle beteiligt. Andererseits waren Stämme, die Shigatoxin 2 allein oder in Kombination mit Shigatoxin 1 bildeten, in 97,1\% Ursache der HUS-Erkrankung (Tab. 2). Die Bedeutung der gelegentlich gleichzeitig oder allein gebildeten Stx-Varianten $2 c$ und $2 e$ ist noch offen.

\section{Diskussion}

Die in unseren Instituten erhobenen bakteriologischen Ergebnisse des Jahres 1997 bestätigen den seit einigen Jahren zu beobachtenden Trend einer Ausweitung der Erregertypen mit einem Rückgang des prozentualen Anteils der Serogruppe $\mathrm{O} 157[4,5]$. Neben den bereits 1996 beschriebenen EHEC der OGruppe 103 trat 1997 der Serovar O145: $\mathrm{H} 28 / \mathrm{H}^{-}$vermehrt auf und war mit $8,7 \%$ dritthäufigster Erreger von HUS-Erkrankungen. Diese Erregerdynamik unterstreicht die Bedeutung einer O-Gruppen-unabhängigen bakteriologischen Diagnostik mit Zielrichtung auf die Shigatoxinbildung mittels phänotypischer (Stx-ELISA, Zytotoxizitätstest) oder genotypischer Methoden (StxPCR). Diesen meist als Suchtest angewandten Verfahren muß die Erregerisolierung mit anschließender Bestätigung folgen; die seit 1997 vorliegenden diagnostischen Empfehlungen von $\mathrm{RKI} / \mathrm{BgVV}$ und DGHM [2] bestätigen diese Forderung. Die Suche nach dem Erreger in Keimgemischen gestalte sich nicht selten mühevoll und zeitaufwendig; ein kürzlich beschriebener ver besserter Vancomycin-Cefixim-Cefsulodin-Blutagar zum Nachweis des EHEC-Hämolysins [8] kann in vielen Fällen hilfreich sein. Bei negativem $\mathrm{Er}$ gebnis auf diesem Nährboden ist allerdings die weitere Suche mittels DNSKoloniehybridisierung oder KolonieImmunoblot unumgänglich.

Die Feststellung der Stx-Typen hat in den vergangenen beiden Jahren zu der bemerkenswerten Beobachtung geführt, $\mathrm{da}$ offensichtlich dem Shigatoxin 2 eine größere pathogenetische Bedeutung für die Entwicklung des HUS zukommt als dem Shigatoxin 1. Die frühzeitige Unterscheidung der Shigatoxintypen im mikrobiologischen Labor ist somit wünschenswert, da hieraus möglicherweise prognostische Schlüsse im Hinblick auf den weiteren Verlauf der EHEC-Infektion beim Patienten gezogen werden können.

EHEC-Stämme im engeren Sinne sind durch den Besitz der chromosomalen Pathogenitätsinsel LEE (Markergen eae) sowie des Virulenzplasmids definiert, auf dem als markantestes Virulenzmerkmal das Gen für das EHECHämolysin (EHEC-bly) lokalisiert ist [9]. Stämme, die nicht das komplette Virulenzmuster besitzen, werden häufig als STEC (»Shigatoxin-bildende E. coli«) oder VTEC ("Verotoxin-bildende E. coli $«$ ) von den EHEC abgetrennt [10]. Für die Humanmedizin ist diese künstliche Unterscheidung aus mehreren Gründen wenig sinnvoll.

1. Wie Tabelle 1 sowie die Beobachtungen der Vorjahre zeigen $[4,5]$, können auch STEC Enteritis und sogar HUS verursachen, wenn auch weniger häufig; STEC sind also durchaus potentielle Krankheitserreger [10].

2. Das künftige Infektionsschutzgesetz sowie die existierenden Länderverordnungen sehen eine Meldepflicht für EHEC, nicht aber für STEC vor. Würde in der medizinisch-mikrobiologischen Diagnostik zwischen beiden Erregergruppen unterschieden, würden u. U. schwer verlaufende Darminfektionen durch STEC als nicht seuchenhygicnisch relevant angesehen. Dies ist vor dem Hintergrund weiterer und in ihrer Bedeutung noch nicht endgültig aufgeklärter Virulenzfaktoren (hitzestabiles Enterotoxin (EAST), Katalase-Peroxidase (KatP), Serinprotease (EspP)) nicht zu verantworten.

3. Der Nachweis des eae ist in der Praxis nur mittels molekularer Methoden möglich und würde bedeuten, dafs diese Methodik bereits Bestandteil der Primärdiagnostik sein müßte. Da im übrigen Stämme unterschiedlicher Virulenz bei allen Krankheitserregern die Regel sind, ist aus humanmedizinischer Sicht kein Grund zu sehen, von der etablierten Bezeichnung EHEC bei Infektionen des Menschen abzuweichen.

\section{Schlußfolgerung}

EHEC haben auch 1997 ihre Fähigkeit zur ungehemmmten Ausbreitung unter 
Beweis gestellt; die ständige Ausweitung der Erregertypen bei menschlichen Erkrankungen belegt dies in überzeugendem Maße. Der kürzlich erfolgte Nachweis der Erreger in migrierenden Vogelarten (Brandgänse, Möwen) [11] oder das Vorkommen Shigatoxin-2-kodierender Bakteriophagen in Abwasser [12] zeigen neue Möglichkeiten der schnellen Verbreitung und damit auch der Übertragungsmöglichkeiten auf den Menschen auf. Während sich bisher mit Recht - die Bemühungen des gesundheitlichen Verbraucherschutzes primär auf tierische Lebensmittel vom Rind konzentrierten (Milch, Fleisch, Rohwurst), werden zukünftig auch pflanzliche Lebensmittel in Betracht gezogen werden müssen, wenn die Kontamination über Oberflächenwasser, Dung oder Vogelkot nicht auszuschlieBen ist.

Besonders nachdenklich stimmt in diesem Zusammenhang die Beobachtung, daß EHEC-Stämme in Pflanzengewebe eindringen und sich damit dem Waschprozeß entziehen können [13]. Es wird somit mehr denn je einer intensiven und kontinuierlichen Ermittlung der Infektionsursachen bei EHEC-Infektionen des Menschen bedürfen, um rechtzeitig Maßnahmen ergreifen und unsere Bevölkerung wirkungsvoll vor diesen $\mathrm{Er}$ regern schützen zu können.

\section{Literatur:}

[1] Robert Koch-Institut und Bundesinstitut für gesundheitlichen Verbraucherschutz und Veterinärmedizin: EHEC-Infektionen - Erkennung, Verhütung, Bekämpfung. Merkblatt für Ärzte, Stand 1996. Bundesgesundbbl. 40, 6 (1997) 210-211.

[2] Robert Koch-Institut: Zum Aufbau einer Surveillance für enterohämorrhagische $\mathrm{E}$. coli (EHEC) in Deutschland. Epidem. Bull. 39/9? (26. September 1997) 269-273.

[3] Huber, H. C., Kugler, R., und Liebl, B.: Infektionen mit enterohämorrhagischen Escherichia coli (EHEC) - Ergebnisse einer epidemiologischen Erhebung in Bayern für den Zeitraum April 1996 bis März 1997. Gesundheitswesen 60 (1998) 159-165.

[4] Bockemühl, J., und Xarch, H.: Zur akruel len Bedeutung der enterohämorrhagischen Escherichia coli (EHEC) in Deutschland (1994-1995). Bundesgesundhbl. 39, 8 (1996) 290-296.

[5] Bockemühl, J., Karch, H., und Tschäpe, H. Infektionen des Menschen durch enterohämorrhagische Escherichia coli (EHEC) in Deutschland, 1996. Bundesgesundhbl. 40, 6 (1997) 194-197.

[6] Prager, R., Reissbrodt, R., Holler, H., Aleksic, S., Gericke, B., Claus, H., Wagner, H., und Tschäpe, H.: Isolierung und Charakterisierung von Shigatoxin-produzierenden E. coliStämmen aus Stuhlproben - Ergebnisse eine Sentinel-Srudie. Bundesgesundhbl. 41, Sonderheft Oktober (1998) 6-13.

[7] Von Hase, U., Pulz, M., und Windorfer, A. EHEC in Niedersachsen, Januar 1995 - Au gust 1997. Niedersächs. Ärzteblatt 11 (1997) 20-23, 38-40.

[8] Lehmacher, A., Meier, H., Aleksic, S., and Bockemühl, J.: Detection of hemolysin vari- ants of Shiga toxin-producing Escherichia coli by PCR and culture on vancomycin-cefiximecefsulodin blood agar. Appl. Environm. Microbiol. 64 (1998) 2449-2453.

[9] Tesh, V. L.: Virulence of enterohaemorrhagic E. coli: role of molecular cross talk. Trends in Microbiol. 6 (1998) 228-233.

[10] Richter, H., Klie, H., Timm, M., Gallien, P., Perlberg, K.-W., Teufel, P., und Protz, D.: Verotoxin-bildende Escherichia coli (VTEC) in Milch, Fleisch und Wurst von Rindern als potentielle enterohämorrhagische Escherichia coli (EHEC). RKI Info II/98 (1998) 3-6.

[11] Wallace, J. S., Cheasty, T., and Jones, K.: Isolation of Vero cytotoxin-producing Escherichia coli O157 from wild birds. J. Appl. Microbiol. 82 (1997) 399-404.

[12] Muniesa, M., and Jofre, J.: Abundance in sewage of bacteriophages that infect Escherichia coli $\mathrm{O} 157: \mathrm{H7}$ and that carry the Shiga toxin 2 gene. Appl. Environm. Microbiol. 64 (1998) 2443-2448.

[13] Iroh, Y., Sugita-Konishi, Y., Kasuga, F., Iwaki, M., Hara-Kudo, Y., Saito, N. Noguchi, Y., Konuma, $\mathrm{H}_{\text {., }}$ and Kumagai, S.: Enterohemorrhagic Escherichia coli O157:H7 present in radish sprouts. Appl. Environm. Microbiol. 64 (1998) 1532-1535.

\section{Anschrift der Verfasser:}

Prof. Dr. Jochen Bockemühl, Hygiene Institut Hamburg, Abr. Bakteriologie, Nationales Referenzzentrum für Salmonellen und andere bakterielle Enteritiserreger des RKI, Marckmannstr. 129a 20539 Hamburg; Prof. Dr. Helge Karch, Institut für Hygiene und Mikrobiologie der Universität, Josef-Schneider-Str. 2, 97080 Würzburg; Prof. Dr. Helmut Tschäpe, Robert Koch-Institut, Bereich Wernigerode, Nationales Referenzzentrum für Salmonellen und andere bakterielle Enteritiserreger des RKI, Burgstr. 37, 38555 Wernigerode 